\title{
Structural Mechanism Analysis of Orderly and Efficient Vesicle Transport by High-resolution Imaging and Fluorescence Tracking
}

Haijiao Xu ${ }^{\dagger}+$, Jing $\mathrm{Gao}^{\dagger}$, Mingjun $\mathrm{Cai}^{\dagger}$, Junling Chen", Qingrong Zhang ${ }^{\dagger}$, Hongru $\mathrm{Li}^{\dagger}$, ", Hongda Wang ${ }^{*}, \dagger, \S, /$

'State Key Laboratory of Electroanalytical Chemistry, Changchun Institute of Applied Chemistry, Chinese Academy of Sciences, Changchun, Jilin 130022, P.R. China

${ }^{\ddagger}$ Graduate University of Chinese Academy of Sciences, Beijing, 100049, P.R. China

${ }^{\S}$ Laboratory for Marine Biology and Biotechnology, Qingdao National Laboratory for Marine Science and Technology, Qingdao, Shandong 266237, P.R. China

"University of Science and Technology of China, Hefei, Anhui 230026, P.R. China

"School of Chemistry and Chemical Engineering, Wuhan University of Science and Technology, Wuhan, Hubei 430081, P.R. China

*Corresponding author. Tel: +86-431-8526 2453; E-mail: hdwang@ciac.ac.cn

\section{Table of contents:}

Figure S1 AFM topographic images of the cell membranes and the Golgi membranes. ....................S-2

Movie S1 The dynamic process of EGFR endocytosis entry into A549 cells ............................. S-2

Movie S2 The dynamic process of endosomes fusion and fission ........................................... S-2

Movie S3 and Movie S4 The dynamic process of endocytic vesicles interaction with the TGN ....... S-2

Movie S5 The interaction of endocytic vesicles with the TGN through the kiss-and-run model....... S-3

Movie S6 and Movie S7 3D colocalization analysis of EGF, EGFR and TGN. .......................... S-3

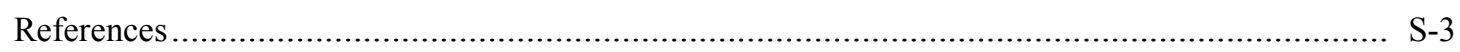



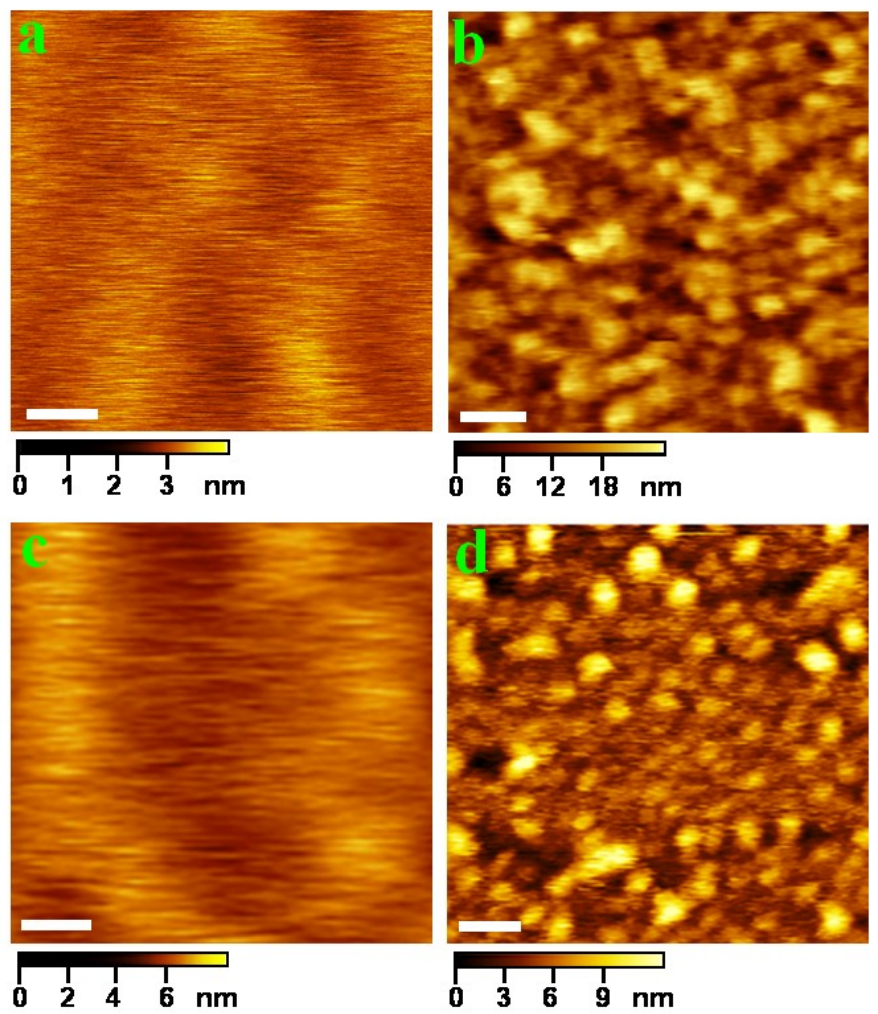

Figure S1. AFM topographic images of the cell membranes and the Golgi membranes. (a) The image of the ectoplasmic side of the A549 cell membrane. (b) The image of the cytoplasmic side of the A549 cell membrane. (c) The image of the outer membrane leaflet of the Golgi membrane. (d) The image of the inner membrane leaflet of the Golgi membrane. ${ }^{1}$ From ref. 1, Figure 3B with permission. Scale bars: $200 \mathrm{~nm}$ in (a) and (b); $100 \mathrm{~nm}$ in (c) and (d).

\section{Movie. S1}

The dynamic process of EGFR endocytosis entry into A549 cells. The movie corresponds to Figure 2a. A549 cells were serum-starved for $48 \mathrm{~h}$ prior to human EGF $(100 \mathrm{ng} / \mathrm{ml})$ stimulation. For receptor internalization experiment, the serum-starved A549 cells were incubated with EGF at $4^{\circ} \mathrm{C}$ for $10 \mathrm{~min}$, then shifted to $37^{\circ} \mathrm{C}$ to trace the dynamic EGFR endocytic process. Acquisition of images occurred at 13 seconds per frame.

\section{Movie. S2}

The dynamic process of endosomes fusion and fission. The movie corresponds to Figure $2 \mathrm{~d}$. The serum-starved A549 cells were incubated with EGF and shifted to $37^{\circ} \mathrm{C}$ to trace EGFR endosome fusion and fission. The area surrounded by the box clearly showed the process of endosomes fusion and fission. Acquisition of images occurred at 20 seconds per frame.

\section{Movie. S3 and Movie. S4}

The dynamic process of endocytic vesicles interaction with the TGN. The Movie S3 correspond to Figure $3 a$. The A549 cells were transiently transfected with mDsRed-Golgi-7 (blue) and EGFP-Rab5 (green) to mark the TGN and early endosome, respectively, and then stimulated with Alex647-EGF (100 ng/ml) (red). Live-cell imaging was used to track the interaction process for $1 \mathrm{~h} 52 \mathrm{~min}$. The marked region in the Movie S3 showed the process of the endocytic vesicles interaction with the TGN. In the Movie S4, the marked region extracted from the Movie S3 indicated the dynamic change of the TGN. Acquisition of images occurred at 1 second per frame. 


\section{Movie. S5}

The interaction of endocytic vesicles with the TGN through the kiss-and-run model. The A549 cells stably expressing EGFP-EGFR (green) were transfected by mDsRed-Golgi-7 (blue). Then, the A549 cells were stimulated with Alex647-EGF (red) and traced by real-time imaging. The image of the cell on the left side of the dynamic image corresponds to Figure $3 b$. Acquisition of images occurred at 30 seconds per frame.

\section{Movie. S6 and Movie. S7}

3D colocalization analysis of EGF, EGFR and TGN. The A549 cells stably expressing EGFP-EGFR (green) were transfected by mDsRed-Golgi-7 (blue) and stimulated with Alex647-EGF (red). Then, the cells were fixed by PFA after endocytosis for $45 \mathrm{~min}$ (Movie S6) and $1 \mathrm{~h}$ (Movie S7). The 3D images were obtained with the Ti-E microscope equipped with a precise motorized stage (PRIOR). Serial Z-stack sectioning was done at $200 \mathrm{~nm}$ intervals. The above images were acquired and processed with the Andor iQ3 image software.

\section{References}

1. Xu, H.J.; Su, W.H.; Cai, M.J.; Jiang, J.G.; Zeng, X.L.; Wang, H.D. The Asymmetrical Structure of Golgi Apparatus Membranes Revealed by In situ Atomic Force Microscope. PLoS One. 2013, 8, e61596. 